\title{
Advanced Classification of Microstructures in EBSD Datasets Using AZtecCrystal
}

Patrick Trimby ${ }^{1}$, Iain Anderson ${ }^{1}$, Kim Larsen $^{1}$, Michael Hjelmstad ${ }^{2}$, Knud Thomsen ${ }^{3}$ and Klaus Mehnert ${ }^{3}$

${ }^{1}$ Oxford Instruments Nanoanalysis, High Wycombe, England, United Kingdom, ${ }^{2}$ Oxford Instruments, Pleasanton, California, United States, ${ }^{3}$ ST Development, Aarhus, Nordjylland, Denmark

Electron backscatter diffraction (EBSD) has become an established characterization tool in a wide range of research fields. EBSD's popularity is driven by its high level of automation, relatively fast acquisition speeds (especially with modern CMOS-based detectors) and, importantly, by the extensive variety of microstructural information that can be extracted from the raw phase and orientation data. Conventionally EBSD indexing involves detection of the Kikuchi band positions using the Hough transform, followed by an indexing process against a set of known phases (and associated look-up tables of Kikuchi band positions and intensities). This requires a certain degree of pre-knowledge about each sample and necessitates enough differences between the atomic and crystallographic structure of each phase so that the Houghbased indexing process can differentiate between the phases. Over the past 20 years a number of technique refinements have improved the EBSD technique's ability to discriminate between phases with similar structures: these include the use of chemical information (typically using energy dispersive X-ray spectrometry - EDS), the use of the relative width of Kikuchi bands and, more recently, the use of patternmatching techniques [1-4]. Recent developments have also indicated the potential of machine learning to determine the crystallography of unknown phases from EBSD patterns [5]. All of these approaches have drawbacks, including limited spatial resolution, limited crystallographic resolution or the requirement for extensive offline reprocessing.

As EBSD data processing tools have become more sophisticated, it has become commonplace to divide a dataset into specific partitions or subsets, enabling individual components of a microstructure to be analyzed independently. This is particularly useful when separating a dataset into components based on grain size or location but is usually less successful when thresholding more continuous parameters such as Kernel Average Misorientation or EBSD pattern quality. In this presentation we introduce new tools within Oxford Instrument's latest EBSD data processing software, AZtecCrystal, that utilize machine learning and a wide selection of microstructural parameters in order to discriminate between constituent parts of a microstructure. These can include phases that are typically indexed using the same crystallographic structure (such as bainite, ferrite and martensite in steels - all usually indexed using a body centered cubic (BCC) structure), or may involve microstructural elements that have been affected by different mechanisms, such as recrystallized and deformed fractions. Within the AZtecCrystal interface, a user can select a limited number of parameters and then train the system to identify regions as a specific class or phase. This recipe can then be applied to similar datasets, enabling a fast and reproducible classification of microstructures into its relevant constituent parts.

In the example shown in Fig. 1, the Kernel Average Misorientation (KAM) map of a deformed and partially recrystallized $\mathrm{Fe} 3 \mathrm{Al}$ microstructure (Fig. 1a) shows clearly the presence of small, recrystallized grains. However, although thresholding the KAM values (Fig. 1b) provides a reasonable first approximation of the 2 main fractions, the local variations in KAM values leads to locally poor results. In contrast AZtecCrystal's phase reclassification tool, using a combination of pattern quality, KAM value and grain information, has successfully separated the microstructure into its 2 major fractions recrystallized (Fig. 1c) and deformed (Fig. 1d). These fractions can then be used to define subsets and their properties (e.g. texture, boundary populations etc.) can be examined independently. Other examples 
of the application of this novel and powerful approach will be presented, including case studies showing the reclassification of bainite, ferrite and martensite structures in steels.

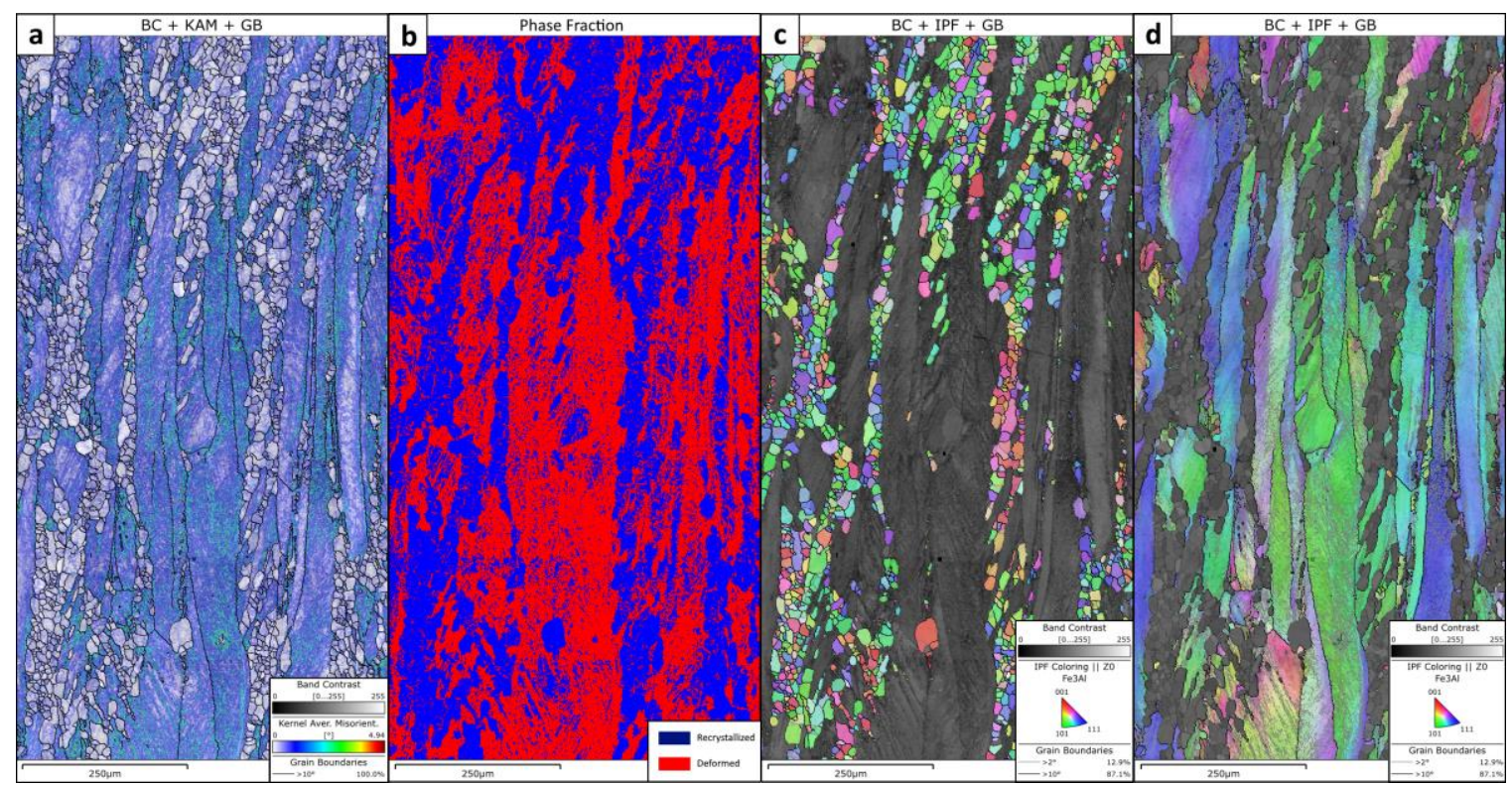

Figure 1. Figure 1. Reclassifying deformed and recrystallized fractions in a Fe3Al EBSD dataset. (a) Kernel Average Misorientation map of the whole dataset. (b) Standard thresholding approach, using KAM values > $0.65 \mathrm{deg}$ : recrystallized shown in blue, deformed in red. Note small areas classed as recrystallized within the deformed grains and vice versa. (c) and (d) Results from AZtecCrystal's machine-learning based reclassification tool, showing orientations (IPF-z direction) of the recrystallized and deformed fractions, respectively with a pattern quality background.

\section{References}

1. Nowell and S. Wright (2004), J. Microsc. 213, 296-305

2. Goulden et al. (2015), Microsc. Microanal 21 (S3), 2033-2034

3. Sitzman et al. (2015), Microsc. Microanal 21 (S3), 2037

4. Winkelmann et al. (2018), Phys. Rev. Mat. 2, 123803

5. Kaufmann et al. (2020), Science 367, 564-568 